\title{
ON THE LAPLACIAN COEFFICIENTS OF TRICYCLIC GRAPHS WITH PRESCRIBED MATCHING NUMBER
}

\author{
Jing LuO, ZhONGXUN ZHU* \\ Department of Mathematics and Statistics \\ South Central University for Nationalities \\ Wuhan 430074, P.R. China \\ AND \\ RUNZE WAN \\ College of Computer, Hubei University of Education \\ Wuhan 430205, P.R. China \\ e-mail: zzxun73@mail.scuec.edu.cn
}

\begin{abstract}
Let $\phi(L(G))=\operatorname{det}(x I-L(G))=\sum_{k=0}^{n}(-1)^{k} c_{k}(G) x^{n-k}$ be the Laplacian characteristic polynomial of $G$. In this paper, we characterize the minimal graphs with the minimum Laplacian coefficients in $\mathscr{G}_{n, n+2}(i)$ (the set of all tricyclic graphs with fixed order $n$ and matching number $i$ ). Furthermore, the graphs with the minimal Laplacian-like energy, which is the sum of square roots of all roots on $\phi(L(G))$, is also determined in $\mathscr{G}_{n, n+2}(i)$.
\end{abstract}

Keywords: Laplacian characteristic polynomial, Laplacian-like energy, tricyclic graph.

2010 Mathematics Subject Classification: 05C12, 05C50.

\section{REFERENCES}

[1] B. Bollobás, Modern Graph Theory (Springer-Verlag, 1998). doi:10.1007/978-1-4612-0619-4

[2] J. Guo, On the second largest Laplacian eigenvalue of trees, Linear Algebra Appl. 404 (2005) 251-261. doi:10.1016/j.laa.2005.02.031

This project is supported by the Foundation of State Ethnic Affairs (14ZNZ023), Natural Science Foundation of Hubei Province (2015CFB405) and Hubei Provincial Department of Education Scientific Research Programs for Youth Project (Q20153003).

* Corresponding author. 
[3] C.-X. He and H.-Y. Shan, On the Laplacian coefficients of bicyclic graphs, Discrete Math. 310 (2010) 3404-3412.

doi:10.1016/j.disc.2010.08.012

[4] A. Ilić, Trees with minimal Laplacian coefficients, Comput. Math. Appl. 59 (2010) $2776-2783$.

doi:10.1016/j.camwa.2010.01.047

[5] S. Li, X. Li and Z. Zhu, On tricyclic graphs with minimal energy, MATCH Commun. Math. Comput. Chem. 59 (2008) 397-419.

[6] B. Mohar, On the Laplacian coefficients of acyclic graphs, Linear Algebra Appl. 722 (2007) 736-741.

doi:10.1016/j.laa.2006.12.005

[7] X. Pai, S. Liu and J. Guo, On the Laplacian coefficients of tricyclic graphs, J. Math. Anal. Appl. 405 (2013) 200-208. doi:10.1016/j.jmaa.2013.03.059

[8] D. Stevanović and A. Ilić, On the Laplacian coefficients of unicyclic graphs, Linear Algebra Appl. 430 (2009) 2290-2300.

doi:10.1016/j.laa.2008.12.006

[9] S. Tan, On the Laplacian coefficients of unicyclic graphs with prescribed matching number, Discrete Math. 311 (2011) 582-594. doi:10.1016/j.disc.2010.12.022

[10] S. Tan, On the Laplacian coefficients and Laplacian-like energy of bicyclic graphs, Linear Multilinear Algebra 60 (2012) 1071-1092. doi:10.1080/03081087.2011.643473

[11] D. Stevanović, Laplacian-like energy of trees, MATCH Commun. Math. Comput. Chem. 61 (2009) 407-417.

Received 27 November 2015

Revised 1 April 2016

Accepted 4 May 2016 